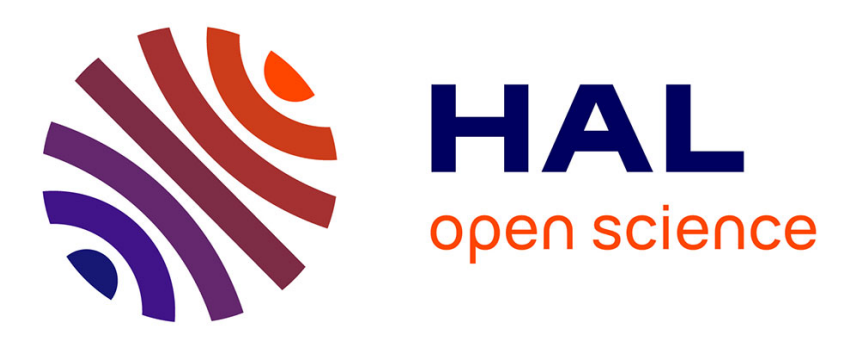

\title{
Technologies and Architectures for Future IP Television Services
}

\author{
Lucile Sassatelli, Marie-Jose Montpetit
}

\section{To cite this version:}

Lucile Sassatelli, Marie-Jose Montpetit. Technologies and Architectures for Future IP Television Services. S. Mohamed Fati, S. Azad, A. K. Pathan. A Comprehensive Guide to IPTV Delivery Networks, Wiley, 2016. hal-01351930

\section{HAL Id: hal-01351930 \\ https://hal.science/hal-01351930}

Submitted on 17 May 2021

HAL is a multi-disciplinary open access archive for the deposit and dissemination of scientific research documents, whether they are published or not. The documents may come from teaching and research institutions in France or abroad, or from public or private research centers.
L'archive ouverte pluridisciplinaire HAL, est destinée au dépôt et à la diffusion de documents scientifiques de niveau recherche, publiés ou non, émanant des établissements d'enseignement et de recherche français ou étrangers, des laboratoires publics ou privés. 


\title{
Chapter: Technologies and Architectures for Future IP Television Services
}

\author{
Contributors: \\ Lucile Sassatelli (Université Côte d'Azur, CNRS, I3S), Marie-José Montpetit
}

\begin{abstract}
This chapter introduces the current trends of evolution of IPTV from several perspectives. First, we review the transformation of the users' expectations and the consequent changes brought to the business opportunities for the different stakeholders willing to benefit from the Internet video usage. The second section of this chapter presents the main architectural overhauls of IPTV led by the converged usage of different video modalities. In particular, we discuss how IPTV and Over-The-Top (OTT) video distribution mutually feed each other to draw the future of IPTV systems, and insist on the current and foreseen collaboration between the stakeholders. The third section focuses on three main technical points in video distribution through the Internet, namely (i) the caching strategies which allow the telecommunication operators (telcos) to scale up their Internet video distribution, (ii) the envisioned solutions to steady and integrate the wireless last-hop into the whole delivery system, and (iii) we open up on the daunting technological challenges arising with Virtual Reality (VR), if VR shall be the upcoming revolution of user experience and ultimate evolution of TV.
\end{abstract}

Introduction

Up until the early 2000s, the IPTV offers mainly followed the linear TV model: the catalog of channels was increased compared to subscription-free (hertzian) and subscription-based (cable) TV services, but the content remained mostly broadcast (where the viewer does not get to choose the show's starting time). To do so, the network providers upgraded their infrastructure to support IP multicast. IPTV providers then started to propose replay services in the mid-2000s, having the shows available for people to watch any time after their airing date. This kind of offers actually developed in parallel of the advent of video streaming services launched by providers not owning network infrastructure but instead relying on the public Internet to reach their consumers, whereby their name of "Over-The-Top" (OTT) services. Several major and smaller video streaming providers hence developed, with various business models based on advertisement (e.g., YouTube) or subscription fees (e.g., Netflix) mainly.

With the advent of ADSL2+, FTTH investments and DOCSIS 3.0 and 3.1, the increase in the clients' access bandwidth in the late 2000s - early 2010s spurred the consumers to subscribe to this kind of unlimited, yet targeted OTT services. An important advantage is their ability to be delivered from any third-party network, contrary to legacy IPTV. The huge leap in mobile access (with $\mathrm{WiFi}, 3 \mathrm{G}$ and 4G) and devices (smartphones and tablets) has intensified this trend, so much so that the legacy TV set and revenue model is being thoroughly disrupted. However, the counterpart of OTT distribution is that the video experience quality depends heavily on the volatile service provided by the Internet, something the IPTV distribution is not sensitive to owing to the control and reservation of the infrastructure, in turn preventing its distribution 
outside the walled garden network of the IPTV provider.

As a result of the above evolutions, IPTV services progress along a path leading to overcome their limitations compared to OTT, so do the OTT services with respect to IPTV. The technological evolutions of both are hence intertwined, enabling an overhaul of the business model of a number of actors involved in these delivery systems. This chapter analyses this future.

\section{The evolutions of users' experience: usage, expectations and reluctances}

The video usage and expectations towards TV services are being disrupted by the new consumption means, be it with mobile devices such as smartphones and tablets, or with network and service offers, such as uncapped mobile data plans and subscription-based video services. This section analyses these major changes in the user behaviors and their impact on the video stakeholders, drawing evolutions towards new revenue models.

\section{a. Broadcast versus Over-The-Top (OTT): towards a spurious opposition}

IPTV lies in the immediate legacy of traditional broadcasting systems designed in the twentieth century. Whether it be radio and television through hertzian waves or plain-old cable television, these systems were and are still meant to reach millions of people with real-time content. Thanks to the undifferentiated content sent to the users, these systems could scale up very well, and their revenue model was based on a combination of subscription and advertisement priced on ratings. IPTV systems have been introduced by fixed telecommunication operators to start to offer a television broadcast service, and by cable operators to broaden their channel offer, leveraging on the Internet access they provide.

However, with the rise of OTT video distribution through new global services like Netflix, Hulu, Amazon Prime and others, a sizable fraction of users have started moving away from traditional TV. In particular, cable subscribers have been increasingly annoyed at the overpriced bundles delivering a great quantity of untargeted content. On the other hand, $90 \%$ of the top 250 shows are available on iTunes or Amazon (Lifehacker, 2014) and the Apple TV reaches ten million units in 2014 (only supporting apps and without any Internet-based live TV service, as of January 2016 where Apple has not formally announced the launching of such service). One may hence argue that the future of user experience does not lie into the broadcast systems anymore, but instead in OTT distribution, as proclaimed by Netflix CEO Reed Hastings foreseeing the death of traditional broadcast by 2030 (Huffington Post, 2014). This clear-cut and somewhat provocative prophecy can be discussed in the light of a number of elements.

First, one of the main widely acknowledged limitations of OTT is in terms of scale when it comes to broadcasting live events (in particular major sport events like the Super Bowl, the Olympics, etc.). To reach millions of even billions of viewers with a high-quality content, multicast IPTV architecture emanating from broadcast systems have the edge thanks to its better use of bandwidth resources.

Second, the infrastructure allowing efficient large-scale and real-time broadcast already exists, be it through cable or airwaves (CSI magazine, 2015).

Finally, the contents desired by the users will always entail a combination of live and not personalized events, fitted to broadcast, with interactive and on-demand shows. 
Even though OTT distribution will keep evolving, it is hence very likely that sensible evolutions of the video landscape will rather involve advanced partnerships of the pipes owners, broadcast industry and content retailers to smartly marry IPTV and OTT architecture, rather than merely wiping out non-OTT systems. The next sections mention announcements of big industry players supporting this claim.

\section{b. The multi-screen multi-device anywhere experience}

With the advent of Subscription-based Video on Demand (SVoD) services along with the manifold of devices to access such content, the watching habits have dramatically changed over the last few years. To exemplify the surge of such services, their UK revenue grew by $56 \%$ over a time span of 12 months, to reach $\$ 417$ million in 2014 , and is expected to total over 11 billion by 2019 (Mintel, 2015). This phenomenon is accompanied by an increasing number of people dropping cable, often referred to as "cord-cutters". In 2014 in the US, Sandvine pinpointed these users for consuming 11-times as much streaming content as other typical users, and accounting for nearly $54 \%$ of total traffic at evening peak hours, while representing only $15 \%$ of all users (Sandvine, 2014). It is expected this share to increase as the young generations of viewers are growing away from the so-called "linear-TV" model, often being dubbed "cordnevers". Additionally, according to (IPTV news, 2016), the number of households without a traditional TV set reached an unprecedented 5 millions in the US, the video content being more and more consumed on laptops and mobile device such as smartphones and tablets.

At the same time however, connected TV devices, in particular SmartTVs, set-top-boxes (STBs) and dongles reached over 200 million sales in 2015 (Strategy Analytics, 2015). As discussed in the first paragraph, the above figures of streaming service subscription and "zero-TV" households do not reflect a radical pushback against traditional broadcaster services, but rather uncover that the use of these gateways to online video content is fueled by the desire of users to control how they watch TV. In particular, the multi-device multi-screen needs of today's consumers are reflected by the increasing number of dongle technologies, such as Miracast or Chromecast, or more recently the French SoftAtHome Universal Cast dongle. This is driven by the desire of users to exploit the full flexibility allowed by the various screens and devices they possess: choosing the screen on which they are going to watch a live premium broadcast or an on-demand show (large TV screen, desktop, screen hooked up to a game console, tablet or even smartphone), while having a unified interface to remotely control and switch channels and screens. This is decisive to the user experience (IPTV News, 2015). As an example, the SoftAtHome Universal Cast dongle allows to do so by additionally supporting multiple in-home casting technologies (Apple Airplay and Google cast).

Finally, legacy IPTV networks are by definition networks owned and managed by the operator providing the subscription, which used to ensure higher quality of video experience compared with OTT. To meet the user's expectation of accessing the content they have subscribed to from anywhere, as they can with OTT, IPTV systems will have to embrace this "anywhere" access, possibly establishing partnerships with other networks (this is detailed in Section III.2). The success of convicted Aereo, a NYC-based company offering such an aggregated experience to its subscribers, illustrates sharply the need yet to be satisfied. Current TV solutions offer STBs which must be hooked up to the screen and multiplied to extend to other screens to switch from live broadcast to on-demand free or SVoD content. Aereo penetrated the market by relieving the user from the hassle of piecing up all systems together, providing them with a seamless multi-screen anywhere experience of watching all cable, broadcast channels and 
VoD content they wanted from where they wanted.

Delivering a high-quality and fully-converged experience to the subscribers wherever they are on whatever screen they want is hence the motto driving the IPTV innovations.

\section{c. Business experiences and inevitable evolutions for the stakeholders}

While the above elements show a demand for still consuming broadcast content as it airs, additionally to free or subscription-based $\mathrm{VoD}$, it also questions the business model on which the major broadcast, cable and content creator companies rely to date. The Aereo adventure, though ended with demise, can be seen as a watershed moment in this regard. Aereo indeed built its business by providing the users with the manifold of the content through virtual antenna deployed in their premises as IP gateways transducing content to re-broadcast it in an aggregated form to their subscribers. Hereby making an attempt on the monopolies of the entitled broadcasters, Aereo has been eventually taken down by a Supreme Court ruling on 20 June14. Nevertheless, its ephemeral success opened the avenue for a rethinking of the way IPTV shall be provided and the revenue opportunities for the legacy broadcasters, whose model based on ads and affiliates, and subscription for cable companies, seems to getting antiquated rapidly in light of the subscription decrease (BGR, 2015).

First, service providers must make the most of the customers' demand for live and public broadcast by embracing IPTV distribution, partnering with TV gateways and accessing those subscribers the way they want again. An example of this realization by some broadcasters is the recent purchase by the UK TV channel Sky of a startup providing OTT deployment to newcoming content providers (CSI Magazine, 2015),(IPTV News, 2014).

Second, cable and telecommunication operators which own the pipes to the users and possibly an IPTV architecture they have already invested in, must depart from their stance as the only entitled to reach users with high-quality video. They instead have to open up to profitable partnerships to make the most of the newcomers to the Internet video market, whose development is hampered by (i) their lack of proprietary infrastructure (Akamai press release 1, 2012),(Akamai press release, 1 2013),(Akamai press release 2, 2012), (Akamai press release 2, 2013),, and (ii) their lack of access to live public TV content (see Apple talks with ESPN, CBS (9to5mac, 2016),(Macworld, 2016)). It is worth noting that the monetization of the telcos' infrastructure to OTT providers, driven by (i), likely falls into the net neutrality problems. The successive FCC rulings on this point (spurred by the Netflix/Comcast/Verizon frictions (Time, 2014)) underline the intricacy of such partnerships in the Internet sphere.

\section{Architectural evolutions of IPTV: toward a smart meld with OTT}

The evolutions of the high-level aspects on video consumers and market discussed in the previous section are driving the architectural changes currently undergone by the IPTV distribution systems. This section presents how IPTV 2.0 is aiming at incorporating all the assets of OTT, while OTT systems in turn look for embracing IPTV key principles to meet the scaling challenge they are facing. While the first section (a) below focuses on the evolutions of legacy IPTV to meet with the OTT-induced requirements of the clients, subsequent sections (b) and (c) 
synthesize how IPTV inspires OTT evolutions, thereby unveiling the cross-fertilization increasingly at work between IPTV and OTT.

\section{a. The main overhauls of IPTV: HTML5, cloudification, software-defined video}

Inheriting from traditional non-IP broadcast systems, IPTV has been turned from the beginning toward delivering high-quality content to masses. On the other side, OTT stems from public Internet Web-like delivery, providing video with native anytime anywhere ability (thanks partly to unicast, see Section II.b), at the expense of hardly supporting mass live broadcast, for which IPTV keeps using multicast delivery to easily scale up. A number of OTT-allowed features are however key to the future of IPTV which cannot overlook them to meet users' expectation.

The first of these OTT features are implied by the support of a multi-screen multi-device experience. That necessitates unifying the delivery framework, which today is achieved by HTML video, specifically W3C-endorsed HTML5 that specifies so-called video element as a standard way to embed a video without the need for additional plugin (for example in a webpage). From a standard and algorithmic point of view, so-called Adaptive Bit Rate (ABR) and the corresponding standard MPEG-DASH (ISO/IEC DIS 23009-1, 2012) enable the video quality to adapt dynamically to the user's and network's environment. The resolution is specific to the screen size, while the video bit rate can be adapted over time to best fit the bandwidth available to the client. More details can be found in recent control-theoretic adaptation logic (Yin et al., 2015). Having such an IP-based video delivery therefore allows for a unified form of content to serve any sort of device (SmartTV, STB, tablets, Web apps, etc.) from any type of access (cable, DSL, cellular, satellite).

The second key OTT feature is offering the access to the unified content from networks not managed by the IPTV provider, to provide the "anywhere" access to the user, by adopting a unicast-based delivery mode not necessitating proprietary infrastructure.

A third key OTT feature IPTV systems must incorporate is the support of VoD (embedding seamlessly legacy OTT services such as Netflix) and advanced Digital Video Recorder (DVR) features, such as catch-up TV, record, pause and rewind. These come natively with OTT services, and IPTV systems do not yet provide such full-fledged capabilities.

The features above are yet seldom offered, or in a quite restrictive mode, to early 2016. To fully embrace them, an important challenge for IPTV providers is indeed to smartly plan and manage the required resources, in terms of storage, computations and network bandwidth. It is hence crucial that these resources can ramp up and down depending on demand variations, so that the provider can make the most of its resource investment while still fully satisfying its customers.

The key enabler of these essential features is for the IPTV providers to rely on cloud resources (additionally to their walled garden), as illustrated in Figure 1. The flexibility and scalability of cloud resources can be fully exploited by so-called Software Defined Video (SDV). SDV allows to depart from specialized video hardware and to leverage the computational power of today's processors and distributed computation. This enables them to deploy, scale and keep up to date video architectures relying on generic equipments seized, specialized and released as demand evolves. Such new solutions are emerging. For example, the Elemental Company (Elemental Technologies, 2016) provides SDV deployment to IPTV providers, and Mware 
Solutions (Mware Solutions, 2016) develops middleware solutions based on HTML5 IPTV standards that can be deployed in the chosen cloud provider as well as in the proprietary infrastructure, targeted at telcos and large Internet Service Providers (ISPs). The cloudification of IPTV services is widely seen as the cornerstone of IPTV 2.0, and is expected to bring the consumers the content and modality of experience they expect, while in turn getting back to a sustainable and profitable revenue model made of a blend of subscriptions, advertisement and partnerships with content creators and providers.

Additionally to the above improvements provided to the customers, SDV allows two important advances for the IPTV provider. First, keeping up with deploying codecs' and display formats' evolutions becomes only a matter of software updates, both at the providers' resources (possibly at a hosting cloud service) and at the clients' app. We mention in passing that the current movements are toward HEVC codec and 4K Ultra HD display format. Second, the right management is a security aspect so far handled differently in OTT and IPTV systems. While DRM support is evolving in OTT and the protection technologies will have to evolve for IPTV to reach out to customers from unmanaged networks, it is envisioned that softwarization and cloudification will be key-enablers for IPTV providers to safely outsource the right management. Enterprises such as (Verimatrix, 2016) are already on this market segment.

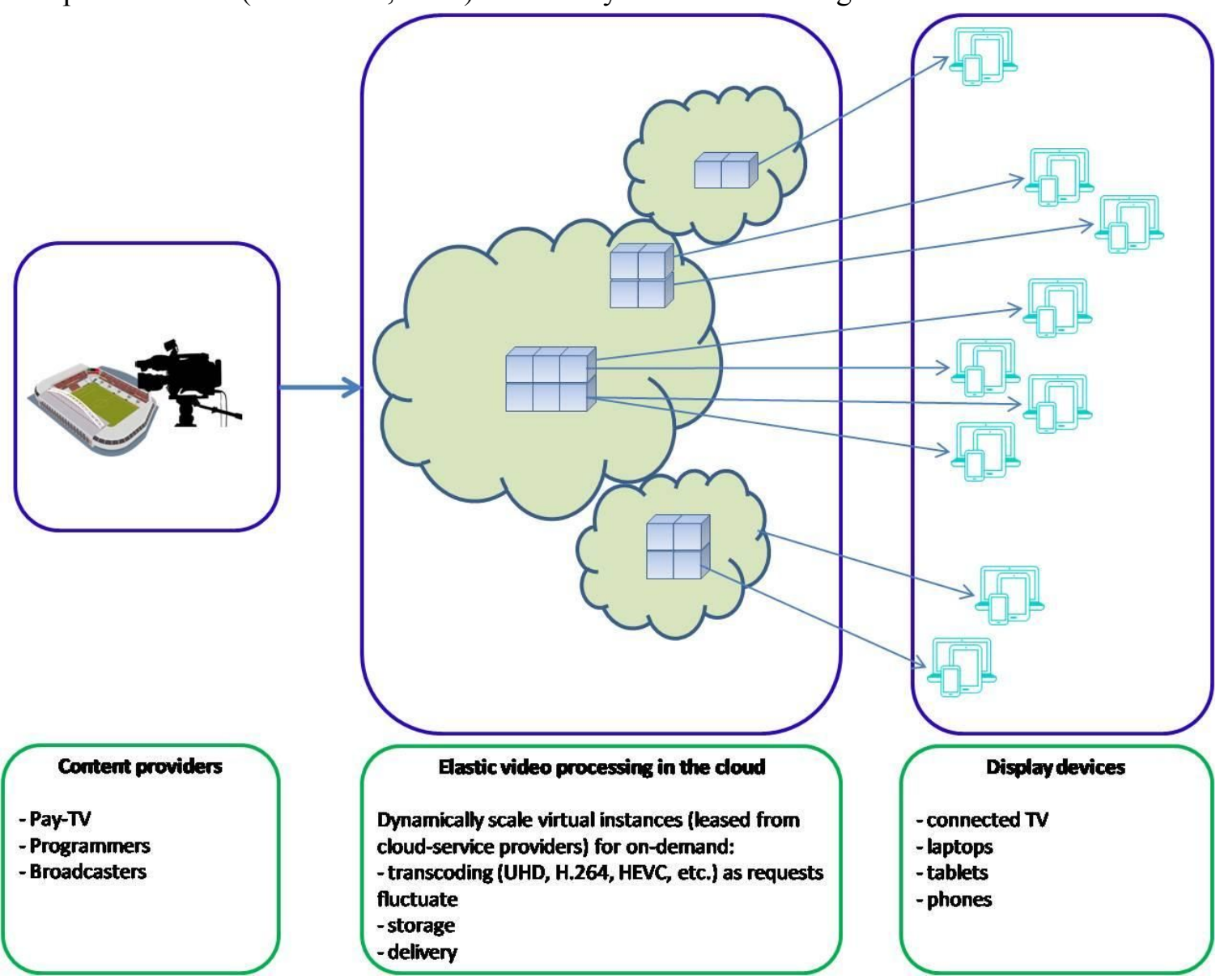

Figure 1: The cloud resources at the service of IPTV providers for quality and cost optimization 


\section{b. Legacy IPTV extending its reach by inspiring OTT evolutions: multicast, caching, Peer-to-Peer (P2P)}

The general architecture of Internet video distribution is sketched in Figures 2 and 3. Within the Internet architecture made of the different tier Internet Service Providers (ISPs), a Content Provider (CP) can send videos to subscribed clients from regular servers (Figure 2). However, when the clients are scattered over several ISPs, to limit the transit costs and increase the number of clients reached with high-quality videos despite the possible congestion, the $\mathrm{CP}$ resorts to a Content Delivery Network (CDN). Amongst the main global CDN companies, we can mention Akamai or Limelight. CDNs have points of presence all over the world, thereby peering with local ISPs easily. The popular content is replicated to be stored close to the users likely to request it, in order to reduce the risk of having insufficient end-to-end bandwidth by lowering the number of hops between the selected server and the client (Figure 3).

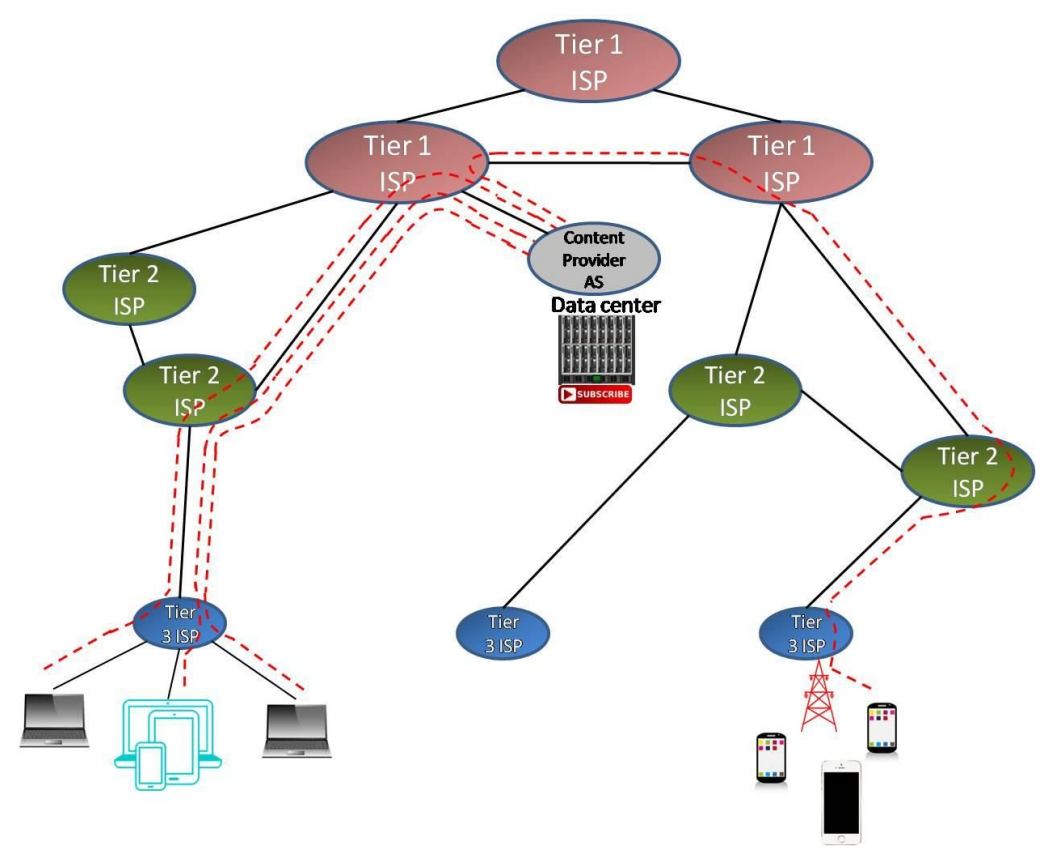

Figure 2: Video delivery over the Internet without any CDN 


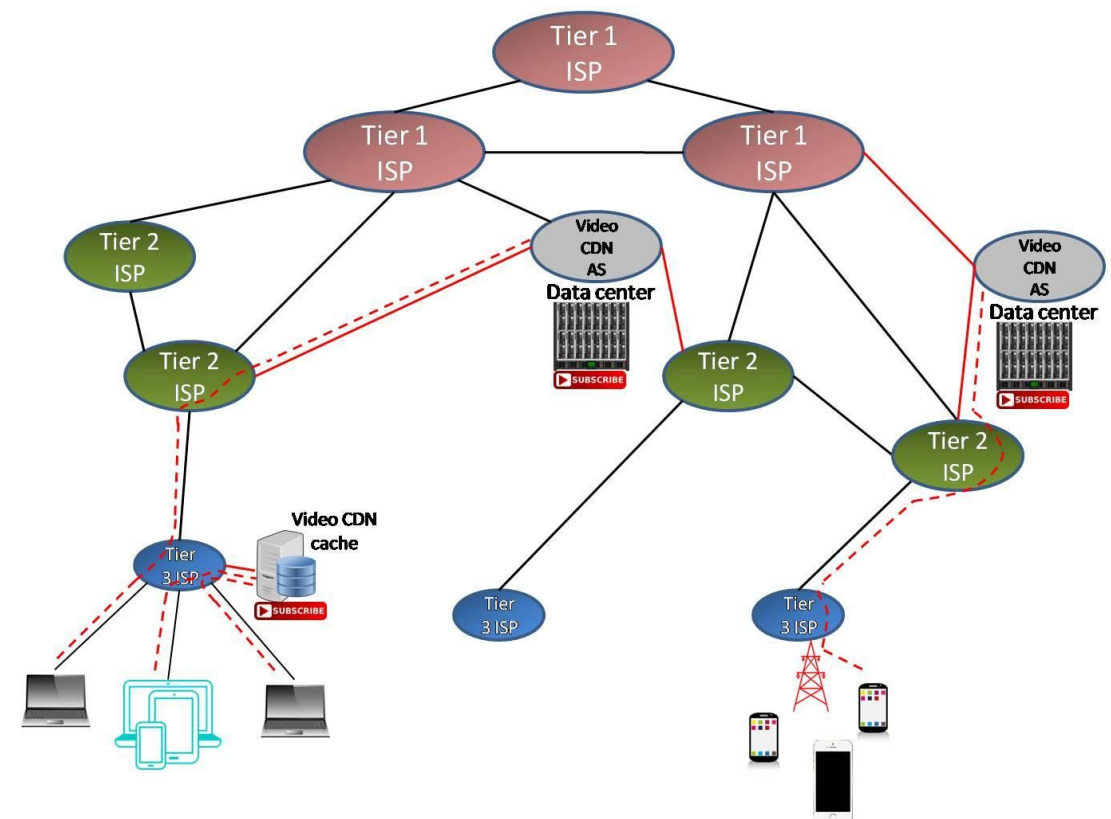

Figure 3: Video delivery over the Internet with the use of a CDN. Peering links and caches close to the users help the delivery.

As live streaming demand increases, the OTT stakeholders (CPs, CDNs, ISPs) have their infrastructure strained, which is a major hurdle for these players to position on the live TV broadcast market. Indeed, Akamai, one of the main CDN companies, recently declared "Akamai forecasts that 500 million viewers will soon be watching prime-time live sports online [needing] 1500Tbps. Today we do 32Tbps" (Munford, 2016).

The default use of unicast connections (or session, these terms are inter-changeable here) in OTT delivery is one of the main causes to this problem. As illustrated in Figure 4, with unicast, a session to the server is established for each client. If several clients request the same content, there will be as many flows with the same content going through in the network, thereby wasting bandwidth resources. On the contrary with multicast, flows are aggregated into a single one, saving bandwidth resources.
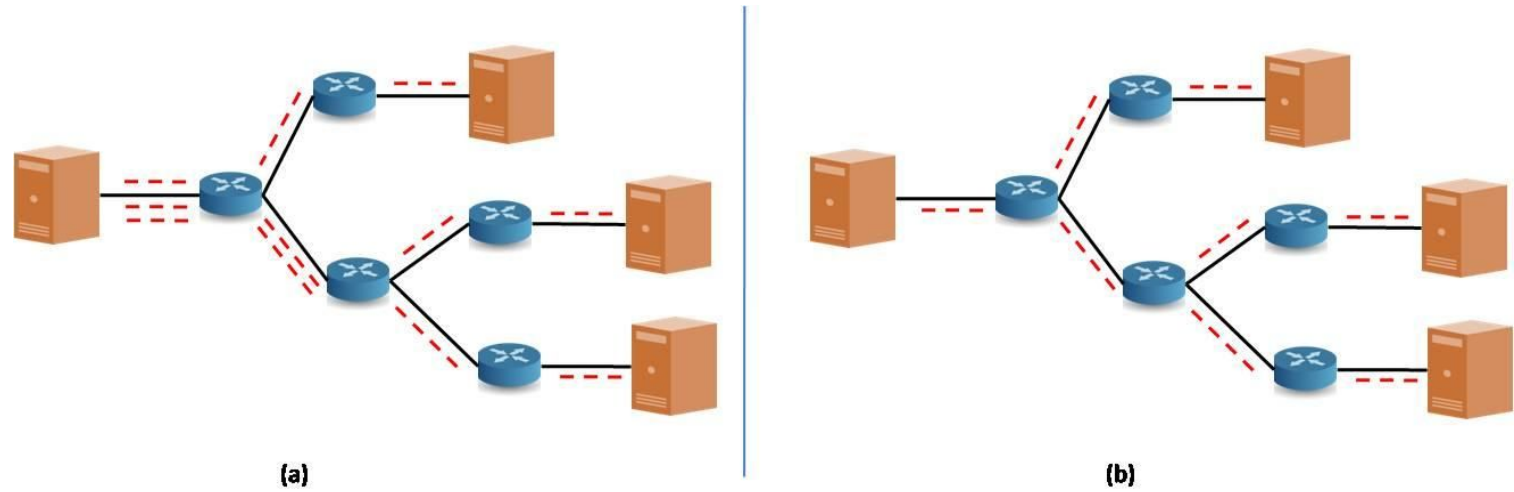

(b)

Figure 4: (a) Between the server (on the left) and the three clients, a unicast session is established for each client. (b) With multicast, flows are aggregated as much as possible to save bandwidth resources by avoiding unecessary replicated transmissions.

As exemplified in the sequel, Akamai, British Telecom (BT) or companies like 
Broadpeak, aim at delivering broadcast-grade TV services across the Internet, including for 4K/UHD video. For VoD distribution too, solutions are sought to alleviate unicast limitations. Toward this goal, the most promising systems currently heavily investigated by the above actors are not brand-new techniques but rather consist in combining revamped architectures that proved efficient in other contexts, namely: IP multicast, P2P sharing and (proactive) caching.

The first pillar of IPTV live distribution is IP multicast, a hot topic for OTT at the 2015 International Broadcasting Convention (Bridge Technologies, 2015),(Streaming Media, 2016). IP multicast is at the core of the legacy IPTV networks, managed by the telcos or ISPs possibly embodying broadcasters. To date though, IP multicast has not been deployed in OTT delivery systems, despite its superiority in resource utilization and video quality for live shows. The first reason for IP multicast not being embraced by ISPs and CDNs earlier is that they have been charging the providers on the aggregate rate used, thereby turning multiple redundant unicast inefficiency into a revenue source (see, e.g., Figure 2). The second reason is that some content rightholders require a unicast session to identify uniquely a user and establish billing this way. While the introduction of CDNs allowed to relieve core networks from the burden of multiple unicast flows from the origin server (owned by the content provider) to the end viewer, multicast is however the main technique able to relieve edge networks from the heavy strain of numerous live streaming unicast flows sent out from the CDN servers to the edge.

By stepping into the OTT delivery market, ISPs and CDNs start entering the privileged group of actors receiving end-user subscription (The Telegraph, 2016). This shift in subscription revenue target suddenly renders IP multicast profitable to these actors: telcos and CDNs have interest in increasing the number of end-users paying and reached with high quality streams at lower infrastructure costs, rather than being paid by the providers based only on the cost incurred on their infrastructure (Streaming Media, 2016). Multicast being forecast as a key-enabler of large-scale live OTT distribution yields another dilemma for the business model: (i) either the telco which has invested into a multicast infrastructure comes into the TV provider market by offering a unified user experience; this is the case of ISPs BT and TalkTalk partnering with the BBC and other channels within the YouView service platform (The Telegraph, 2016), or (ii) the telco's multicast capabilities are leveraged (Broadpeak, 2016),(Akamai, 2016),(Bumgardner, 2015) and the telco can also rent its multicast spectrum to CPs or CDNs. This is a first element revealing an indisputable convergence of large-scale OTT systems toward IPTV founding principles.

The IP multicast momentum for broadcast-quality video is supported by the unicast/multicast hybrid solutions based on Automatic Multicast without explicit Tunnels (AMT) (Octoshape, 2012),(Bumgardner, 2015). AMT has been introduced by Cisco and Octoshape companies. As an indicator of the expected weight of IP multicast into future unified streaming systems, Octoshape has been acquired by Akamai in early 2015. A few months later, Akamai has rolled out its Infinite Edge Solution on February 2016, embracing AMT, and has revealed to intensely negotiate with ISPs to get access to their multicasting resources (Akamai, 2016). AMT is described in more details in the next paragraph. A blunter example of how mere public Internet resources are not sufficient for large-scale OTT delivery (even for stored/non-live VoD) is the recent deal between the OTT giant Netflix and ISPs Comcast and Verizon to lay "data highways" (Time, 2014).

The principle of AMT is to perform unicast aggregation as much as possible, thereby getting back the gains of multicast wherever possible in the different network segments between the video servers and the clients. Figures 5 and 6 represent the AMT principle. The AMT 
architecture is composed of so-called AMT Gateways and AMT Relays (Octoshape, 2012). An AMT Gateway may be a home router or a host (STB, gaming console, PC, etc.), and is in charge of contacting an AMT Relay to initiate the multicast connection. AMT Relays are usually routers at the multicast/unicast boundaries. AMT is a hybrid solution in that it allows to reach any client, multicast and non-multicast enabled alike, while leveraging multicast capabilities wherever available on the end-to-end path and resorting to unicast on the minimum number of links, instead of falling back into unicast on the whole path between the server and the client if the latter is not multicast-enabled. The Broadpeek solution NanoCDN allowing for Multicast ABR (M-ABR) builds on a similar principle with so-called Broadpeak agents in the client premises.

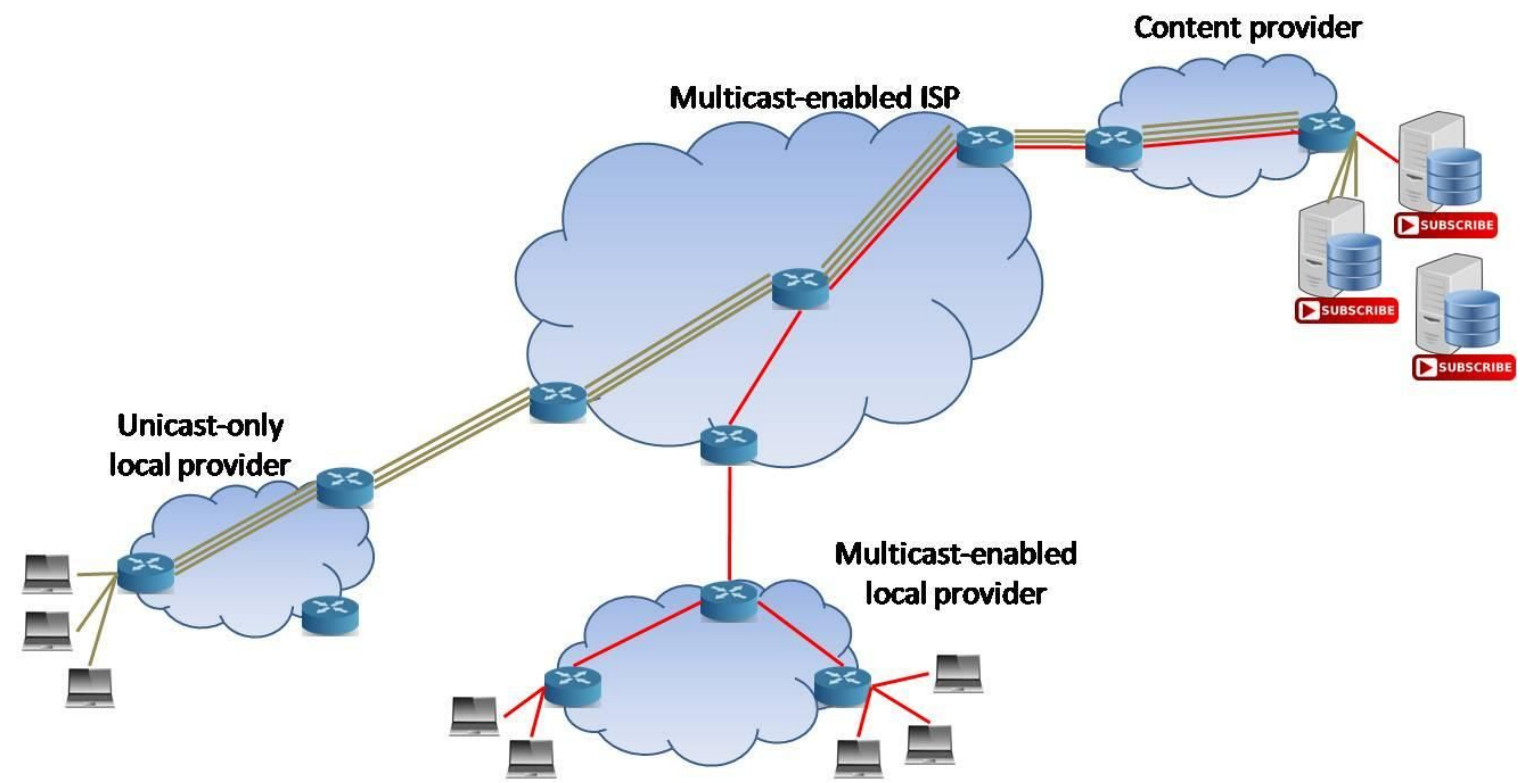

Figure 5: Even though $\mathrm{CP}$ and core network are multicast-enabled, many edge networks remain unicast-only, as the one on the bottom left. Multicast in the global Internet is an all-or-nothing solution: if at least one network of the path is not multicast-enabled, a unicast connection is used end-to-end.

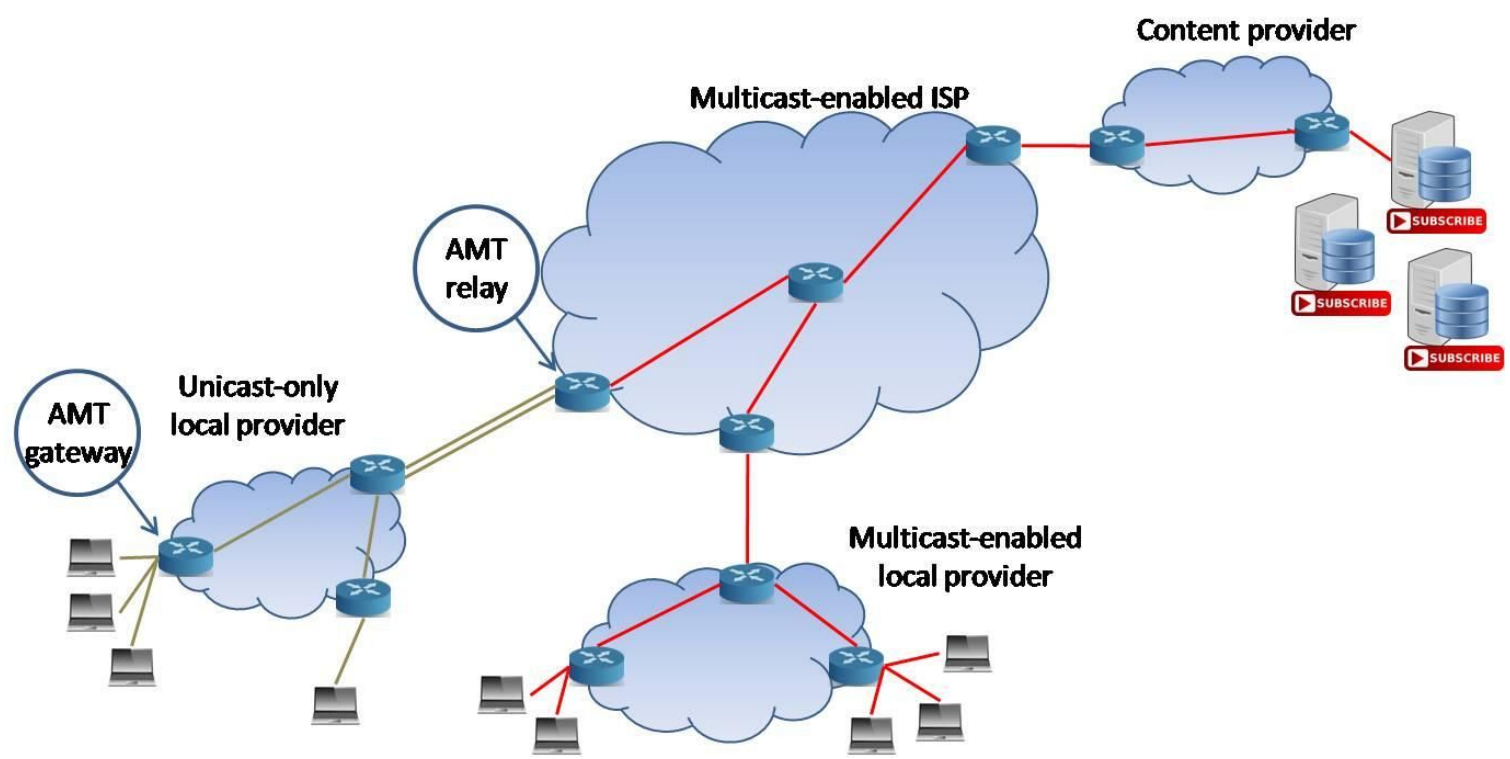

Figure 6: The negotiation between the AMT gateway and AMT relay enables the aggregation of the flows. 
The AMT relay replicates to downstream receivers, adding a unicast header.

A stark example of such advanced integration is the UK ISP and telco BT, which has brought together the revenue models of a regular telco, a CDN and a content provider. By setting up and taking part into a leading-edge consortium of telcos (BT and Talk Talk), solution provider (Arquiva) and broadcasters (TV channels ITV and Channel 5), BT has been able to leverage on the common STB YouView and to build up its own in-network CDN. Each new customer or customer's activity (subscription upgrade) hence directly benefits BT which has succeeded, thanks to smart technical choices and partnership, to truly leverage OTT streaming. BT thereby placed itself in the market of an actual TV cable provider, with a wider offer and unified user experience of a manifold of legacy broadcast and SVoD content all brought together in one place (Streaming Media, 2016). Let us mention that cable ISPs, such as Comcast (US) and Virgin (UK), also make the move to IP multicast towards their subscriber by investing into an architecture upgrade to DOCSIS, the new cable interface specification.

The second heavily explored trail is in-home proactive caching for VoD content. In order to overcome the drop in resource availability (due to volatile events such as flash-crowds) and to better spread the load over time, pre-positioning content in off-peak hours directly into the customer's equipment is closely investigated. This is envisioned for content expected to be watched with a high probability, such as subsequent episodes of a series the user has been watching, or premiers and trailers. Akamai has disclosed to have pre-positioning under the radar (Munford, 2016). A first proof-of-concept had been presented at CES 2014 based on Qualcomm IPQ home gateway and Akamai's client software allowing pre-positioning of content. The concerns on right management and content access are however considered carefully before deploying this kind of system. The NanoCDN solution of the Broadpeak company leverages the home network equipment's storage capability to place such content during the night, additionally employing multicast to do so (Broadpeak, 2016).

The third main investigated avenue to both leverage the in-home caching capabilities and the locality of content popularity (Leconte et al., 2016) is P2P assistance. Along multicasting and pre-positioning, Akamai envisions peer-assistance to $\mathrm{CDN}$ as the third lever in sustaining a large-scale broadcast-like delivery of IP streaming, all the more with WebRTC-enabled interoperability, strongly supported by Google or Apple (WebRTC project, 2016). A large-scale simulation study involving a few millions users has been carried out in 2015 by the BBC and BT (Karamshuk et al., 2015). The Streamroot startup has been recently awarded in this same realm (Streamroot, 2016). The principle, challenges and preliminary results are detailed in Section III.1.

\section{c. Coordinating the OTT delivery entities to enforce IPTV-like Quality of Experience: collaboration between ISPs, CDNs and content providers}

Extending and migrating IPTV services to OTT distribution for the reasons exposed in Section II.a, is conditioned on the ability of OTT to render sufficient quality and reliability, ensuring user/subscriber retention. Video quality is assessed through the user-perceived experience, with so-called Quality of Experience (QoE). Despite the intrinsic subjectivity of such experience, the multimedia community has agreed on three most important metrics for ABR 
(Adaptive Bit Rate) video, as defined in (Dobrian et al., 2011) (Sec. 2.3 therein): the stalls (number and duration), the average bit rate and the instability.

The resulting OTT delivery systems are growing more and more complex owing to the decisions made at the different stakeholders: content provider, CDN, crossed ISPs and client platform. So far, there is little coordination between these different factors in the decision making. The decisions taken at one level are mostly uninformed about the state of the other level. The very meaning of "Over-The-Top" reflects this state of affairs. Specifically, application providers have devised complex workarounds and try to infer, from the clients' feedback, their own statistics about their CDNs and ISPs network states and the features of the clients connected to each ISP. As well, the client side attempts to predict the network state without explicit network feedback. The ISP network has in turn no lever as to how to bend the decisions taken by the cloud system or by the distributed video rate decisions at the edge applications. That is why a number of solutions enabling explicit collaboration are emerging at different standardization bodies (MPEG DASH-SAND, IETF RTCWeb, RMCAT working groups) and new solutions aim at moving the control entirely to a centralized entity (Ganjam et al., 2015), (Georgopoulos et al., 2013). In particular, new control planes' capabilities for ISPs, such as Software-Defined Networking (SDN) coupled with Network Function Virtualization (NFV), along with the emergence of "big data" platforms, are seen as further enablers of a comprehensive cooperation. Such cooperation aims at supporting high-quality, reliable and massive video delivery, which would not correspond anymore to "Over-The-Top" delivery, but to a truly converged unified and managed IP-video delivery.

Amongst the different group or individual proposals (e.g., (Georgopoulos et al., 2013)) for such fully cooperative delivery, we briefly describe a representative framework proposal, led in particular by Databricks and Conviva companies in (Jiang et al., 2014). Two entities, named application providers (AppPs) and infrastructure providers (InfPs) are distinguished. Each entity is made of different subsystems, e.g., client and origin servers for AppPs, and CDNs and ISPs for InfPs, though CDN may traditionally be envisioned pertaining to the application overlay. The distinction may fade for major players such as Google (YouTube) and Amazon. The architecture is made of interfaces between AppPs and InfPs. AppPs can inform InfPs about the client's QoE at different time and space scale, with big data analytics and real-time processing to come up with meaningful and synthetic information representation. InfPs in turn informs AppPs about, e.g., the level of congestion, the control decisions or its peering points.

If such an advanced cooperation is not yet effective, other solutions halfway in this trend are being increasingly adopted. For example, Conviva Company offers solutions to content owners, broadcasters and operators to maximize their subscribers' engagement through smart managements of QoE. Their clients are major media companies, broadcasters, operators and video sites such as Vevo, ESPN, HBO, Sky, BT, France Televisions or Telefonica. One of their flagship solutions is an "Internet-scale control plane for video quality optimization" named C3 (Ganjam et al., 2015). C3 is targeted to handle tens of millions clients, with tens to hundreds of thousands arrivals per minute at peak hours. The video requested are those from the above video providers who rent $\mathrm{CDN}$ hosting from $\mathrm{CDN}$ providers (such as Limelight, Akamai, etc.). Given the CDN infrastructure rented by the provider and the video quality measures it gets, the goal of the centralized control plane is to select the $\mathrm{CDN}$ and the video bitrate to serve the viewing client with. The $\mathrm{C} 3$ controller does not control the CDN distribution logic (cluster and server choices), let alone ISPs, but rather adds a management layer on top of the available delivery ecosystem. A first challenge to get the quality data from the viewers is the heterogeneity of the clients' devices. In order not to impose an overhaul to the client applications, a thin sensing/actuation layer 
exporting raw quality measures has been devised to be seamlessly integrated to existing apps. The second challenge is leveraging on the mass of measures of quality available, and their relative freshness. This is addressed by a split control plane. First a coarse-grained global model layer builds on the stability (on the order of minutes) of the CDN ranking in video quality for each Autonomous System where the viewers reside. A fine-grained per client decision layer then selects the video bitrate for the specific client based both on the former coarse-grained (in time and space) statistics and on the individual up-to-date client state.

\section{Technical focus}

As aforementioned, a number of tools are envisioned by the content providers, CDNs and ISPs to scale up live and Video on Demand (VoD) streaming distribution while delivering a broadcast-like quality despite the uncertainty of the Internet. These tools are specifically IP multicast, peer-assistance to CDN delivery, caching and content pre-positioning.

In the first subsection below, we detail the recent studies and upcoming solutions for legacy CPs and CDNs to leverage P2P assistance in VoD distribution. We will bring up Information Centric Networking (ICN) in this regard.

As video usage through mobile devices amplifies, the last transmission hop at the client side is increasingly wireless, whether it be a cellular or a WiFi channel. Despite link-level retransmissions, it has been shown that the wireless channel impacts hugely the quality of experience perceived by the client. In particular, even in WiFi home environment, which can be reckoned as static, the deployment of multi-screen distribution beyond the set top box wired to the wall, is still a challenge. The second subsection deals with in-home video over WiFi challenges.

To finish, we will discuss the advent of $4 \mathrm{~K}$, multi-view experience and Virtual Reality (VR), highly liable to render the current distribution inoperative, hence calling for a radical change of IP video distribution infrastructure, supported by an achieved integration of all technology elements above to truly bring the needed overhaul.

\section{a. P2P assistance to CDNs, Caching and Information-Centric Networking}

The principle of $\mathrm{P} 2 \mathrm{P}$ assistance to $\mathrm{CDN}$ is depicted in Figure 7. While statistics (Sandvine, 2014) show that P2P file sharing steadily decreases since 2010 owing to the surge of HTTP-based content consumption (and sharing, see, e.g., the shutdown Megaupload website), P2P has been under the radar of CDNs to improve their distribution efficiency. For example, the Akamai Netsession application (Zhao et al., 2013) has been deployed worldwide to offload traffic from the CDN servers. While other P2P-only or hybrid P2P/CDN solutions have been deployed until 2014, they all showed decreased gains when the user traffic was constrained within a same ISP, owing to the low participation or high churn rate (Balachandran et al., 2013). However, ISP friendliness, i.e., keeping the P2P sharing between the users of the same ISP, may be deemed inevitable so as not to incur additional transit costs to their ISPs. Beside (i) ISP friendliness, on-demand video streaming exhibits the following features which may hinder gains from P2P sharing assistance to $\mathrm{CDN}$ : (ii) as $\mathrm{ABR}$ is used, different video bit rates are requested by the user, restricting the number of users which can serve a given one, (iii) the streaming concept imposes 
synchronicity of demands, which shall not be the case with on-demand stored streaming or with DVR commands (pause, fast forward and backward), and (iv) as for regular P2P systems, user participation is determining (e.g., in Akamai Netsession, only 30\% of users have enabled P2P participation (Zhao et al., 2013)).

Remarking that the preponderant share of IP peak traffic corresponds to long-duration shows, Karamshuk et al. in (Karamshuk et al., 2015) investigated whether such feature can mitigate asynchronicity and low participation enough so that $\mathrm{P} 2 \mathrm{P}$ can still bring gains to $\mathrm{CDN}$ in spite of the constraints (i)-(iv) above. To do so, they have carried out extended simulations on a one-month trace of 16 million BBC iPlayer (OTT show replay service) sessions from the greater London area. Gains are quantified as ratio between traffic served by peers and total consumed traffic. The important findings are that with ISP friendliness enforced, only $10 \%$ of peers participating, and different video bit rates available, the gains can still reach $80 \%$. By looking at the impact of users leaving the system before they quit viewing the video (which may happens if the user is able to download the whole content in a shorter time than the video duration), the authors identified that this can cause gains to drop from $80 \%$ to $30 \%$. The "online while watching" assumption is hence crucial to P2P assistance efficiency. Finally, having the peers to store the last five contents they have been watching increases the swarm capacity by a factor of 10 .

Very recently, the Streamroot company has proposed P2P assistance for live video streaming (Streamroot, 2016), based on a P2P proprietary module and WebRTC for a seamless integration in existing HTML5 video players.

The concept of P2P assistance for video streaming naturally relates to the paradigm of Information Centric Networking (ICN) (Xylomenos et al., 2014), which is part of the research activities of the Future Internet Architecture community. ICN trades the concept of host-based communication (where one IP client tries to reach one specific IP server) for the concept of content-based communication, where only the content name is requested to the network, instead of an address. The network equipments have inherent caching capabilities, allowing to store the content ideally close to the user, to improve and scale performance. A client then sends out Interest messages, and when a router/cache holding the desired content receives such message, it forwards the data back to the client following the forward path. By construction, ICN natively supports in-network caching, (multi-source) multicast and multi-path routing, making it very appealing to scale high-quality video distribution. A recent Internet Research Task Force (IRTF) group has started designing pull-based Dynamic Adaptive Streaming (DAS, no HAS as non-HTTP) in ICN (Lederer et al., 2015).

The performance of DAS in ICN has been investigated very recently by Rainer et al. in (Rainer et al., 2016), for different message forwarding and video quality adaptation strategies. In particular, the authors considered H.264/SVC instead of H.264/MPEG-4 AVC video coding. SVC stands for Scalable Video Coding. While H.264/MPEG-4 AVC is the adopted coding in DASH and allows to have independent representations of the content (each encoded at a different rate), H.264/SVC provides a layered representation where obtained quality is a function of the number of received layers (coarse-grained to fine-grained information). SVC therefore allows a client which would have fetched a given quality of a video segment and happens to experience a hike in its available bandwidth, to fetch additional layers to add them to the already obtained base layer to improve the quality. The same situation with AVC would require discarding the first obtained representation to download the higher quality packets. SVC has not been adopted in MPEG DASH (standardized with H.264/AVC), but is instead used by a number of video conferencing tools provided by manufacturers such as Vidyo, Polycom or Radaya. The reason for SVC not to 
be used in stored $\mathrm{VoD}$ is its additional storage cost and processing complexity (outbalanced by its advantages for video conferencing). However, owing to the ICN feature where a client may be served by different caches, the authors of (Rainer et al., 2016) use SVC with MPEG-DASH to increase the hit ratio. Running simulations on different topologies and comparing with optimal results considering unlimited multi-path routing or caching, the conclusion is that the current sub-optimality of HTTP streaming (based on IP) comes from the lack of multi-path support, and that current ICN solutions are still far from optimal where caching is available at each network component.

Complementarily, (Yu et al., 2015) considered centralized control of caching (with SDN) to perform intelligent DASH/AVC representation pre-fetching based on the (ISP's) network load in order to smooth out traffic peaks which drive the network dimensioning and planning.

Although the above works are examples that the ICN concept is a promising solution to scale up high-quality flexible IP video delivery, they also show that the current solutions are highly immature and a lot of efforts remains to be done. Questions in particular are: which level of centralized control is realistic and beneficial in ICN, which kind of information to share between network entities and end points, in particular how to adapt to client's bandwidth variations when considering caching, pre-fetching and limited transcoding resources, and how to deal with function placement problems.

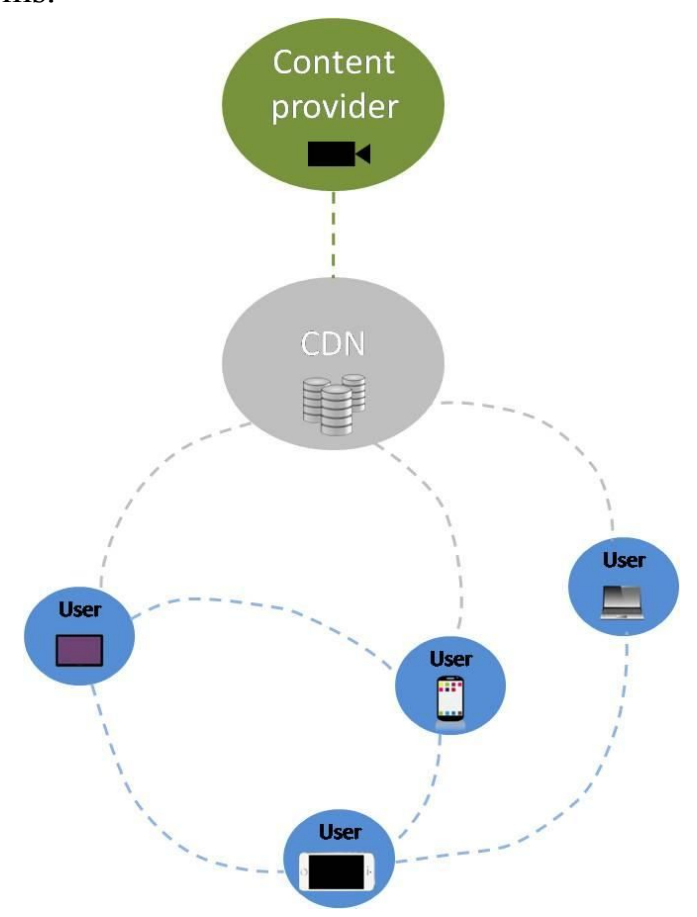

Figure 7: Principle of P2P assistance to CDN delivery

b. The wireless video challenge: in-home WiFi and offloading of cellular networks

In-home WiFi is an essential component of daily video consumption, but high-quality video delivery over this medium remains challenging, as we shall see.

Outdoor WiFi is more marginal, but is foreseen to be a major player in offloading and improving mobile networks. Data offloading through WiFi is in particular included in the LTE architecture (ETSI, 2016), though the main LTE/WiFi offloaded traffic share corresponds to 
delay-tolerant traffic (software updates, file sharing synchronization, etc.) postponed until the mobile device moves and gets a WiFi connection (Lee et al., 2010). In the framework of HetNets (Heterogeneous Networks (Ericsson, 2011)), which aim at bringing together networks of different technologies (LTE, WiFi, Bluetooth, etc.) and reach (macro-cells, femto-cells, pico-cells), new solutions are being proposed. For instance, (Birdstep Technologies, 2016) combines cellular and WiFi to target maximizing coverage with the highest quality connection for the user, while managing operator costs. WiFi access points are often connected to hybrid fiber-coax over DOCSIS or ADSL (Free, 2016). An example of the latter is the case of the French network operator Free where the WiFi APs pertain to the mobile network operator itself. Indeed, Free is a major French provider of DSL (with WiFi routers) and mobile accesses. Partnerships between cable and mobile operators may be envisioned, though a general framework for seamless integration between licensed and unlicensed spectrum access is still actively under study in the HetNets and 5G framework (RCR Wireless News, 2015), (Akhtar et al., 2016).

Inside the home with a dedicated WiFi network, service providers are generally reluctant to guarantee video distribution beyond the cord-tethered STB. Indeed, it has been shown that, despite the large nominal bandwidth available and the seemingly static environment, construction characteristics and intermittent interference (e.g., see (Kim et al., 2013), Fig. 1 therein) can lead to jolting video incurring harsh user dissatisfaction blamed on the STB provider. We mention below the main solutions, likely to integrate upcoming systems, if not yet partially present.

First, some advanced flow scheduling can be performed at the WiFi access point, so as to give video flows the required priority, while not starving the other flows and ensuring medium utilization and fairness (avoiding the well-known head-of-line blocking problem). Second, as Self Organizing Network (SON) has been defined in the LTE (E-UTRAN) standard to smartly handle heavy network loads, one can envision that a counterpart may be beneficial in home WiFi subjected to heavy (4K and beyond) highly-sensitive traffic and difficult network conditions. SDN for indoor WiFi may play this role.

A number of other proposals also build on the idea of adding Forward Error Correction (FEC) coded packets to the initial transmissions, the rationale being that the residual loss rate at the transport layer (after layer-2 corrections), due to the last link being wireless, can often be as high as 5\% (Kim et al., 2013). For example, Coded TCP (Kim et al., 2013) is designed to behave as legacy TCP on wired paths (reducing source rate upon detection of a loss attributed to a buffer drop), but allows to anticipate on wireless losses by sending the right amount of redundancy. Another set of so-called streaming codes (Karzand et al., 2016), currently under active development, aims at generating periodic redundancy within the video stream itself, allowing to lower the loss recovery delay even more.

Another established observation (Esteban et al., 2012) is that the TCP transport of video streams for $\mathrm{VoD}$ or RTC applications entails a number of problems (e.g., latency due to connection establishment, poor performance of unbundled connections - one connection per webpage object with HTTP/1.1, packet loss due to sending bursts). That is why UDP-based protocols have been devised to more efficiently transport this kind of traffic. While UDP is used, Congestion Control (CC), otherwise enforced by TCP, is still needed. Amongst the numerous proposals under considerations at the IETF working groups RTCWeb, RMCAT and TAPS, QUIC (Quick Internet UDP Connections, by Google) is gaining momentum and currently supports half of the communications between Google Chrome and Google servers (Iyengar and Swett, 2015). Other proposals for real-time flows include, e.g., Google CC or Network Assisted Dynamic Adaptation (NADA) CC algorithm (Lundin et al., 2013) which build on RTP and RTCP signaling to let the source compute more precisely the rate it can use, unlike legacy TCP which only allows it to infer the network state of congestion by packet loss detection. In particular, QUIC tailors 
Cubic CC additionally to integrating the key ingredients of HTTP/2.0 such as connections bundling into streams (TCP Cubic is the legacy TCP deployed in Linux, used by more than 65\% of the servers in the Internet (Yang et al., 2014)). Furthermore, QUIC enables acknowledgment disambiguation by its own sequence numbering, as well as FEC to retrieve from packet losses without having the receiver necessarily ask for retransmission.

\section{Open research issues}

While the above solutions either start appearing for Internet video/television delivery, or are likely to appear in the upcoming years, a tremendous revolution in the user's experience lies ahead: Virtual Reality. Early 2016, a number of major events have meaningfully focused on VR: CES 2016, and Google I/O 2016 is announced to be a milestone in the still early outset of VR.

As an intermediate step between legacy video experience (with flat or 3D images, small definition or $4 \mathrm{~K}$ ) and VR, we can mention so-called interactive broadcast experience, which provides the user with the possibility to decide from which camera he wants to view the show/event. Recently, Free-Viewpoint Television (FTV) (Chen et al., 2016) has been proposed as an even more immersive experience where the user can decide an arbitrary viewpoint by having different camera streams combined at the player. In particular, the network challenges of doing so for live delivery and sizable audience are investigated in (Chen et al., 2016).

Some systems currently available early 2016 are tagged "VR". For example, Oculus Rift and HTC Vive headsets are able to render $4 \mathrm{~K} / 360^{\circ}$-captured/possibly in $3 \mathrm{D}$, videos, and require consequent computational power to do so (CNET, 2016). Google provides a mere cardboard box to slide a smartphone in and watch a $360^{\circ}$ flat video a inch away from the eyes with magnifying lenses. There is much debate as to whether this kind of low-cost VR should actually be pegged as VR (Ciscso Newsroom, 2016).

The very sense of VR indeed refers to making the content appear as reality to the human user. A precise definition of what we, as humans, perceive as "reality" is hence necessary to assess whether the system truly provides VR. Recently, Bo Begole from Huawei made such a detailed analysis, for the view sense only, though all the other senses are involved, in particular earring that we already know to remotely stimulate as the eye (Begole, 2016). Taking into account our fovea's dot detection capability, field of view and eye's shift speed, he comes up with a bit rate of $5.2 \mathrm{Gbps}$ (considering best current codec H.265 HEVC). Even a hundred-time lower bit rate would be unsustainable by most broadband accesses, as defined by the US FCC at 25Mbps (and storage may be an issue too). VR hence poses tremendous and daunting challenges to delivery through network, and these challenges are open avenues for most innovative research.

\section{References}

9to5mac, 2016. ESPN president says Apple 'frustrated' over building TV service, expects new packages in 2016. [online] Available at: <http://tinyurl.com/hddneg3 > [Accessed 20 June 2016].

Akamai press release 1, 2012. Akamai and AT\&T Forge Global Strategic Alliance to Provide Content Delivery Network Solutions. [online] Available at: <http://tinyurl.com/goort83> [Accessed 20 June 2016]. 
Akamai press release 1, 2013. KT and Akamai Expand Strategic Partnership. [online] Available at: $<$ http://tinyurl.com/zlozpeb $>$ [Accessed 20 June 2016].

Akamai press release 2, 2012. Orange and Akamai form Content Delivery Strategic Alliance. [online] Available at: <http://tinyurl.com/z6j3uh6> [Accessed 20 June 2016].

Akamai press release 2, 2013. Swisscom and Akamai Enter Into a Strategic Partnership. [online] Available at: $<$ http://tinyurl.com/h7bt5m8 $>$ [Accessed 20 June 2016].

Akamai, 2016. Infinite Edge Solution. [online] Available at: <http://tinyurl.com/jpuunr9> [Accessed 20 June 2016].

Akhtar, A. M.; Wang, X. and Hanzo, L., 2016. Synergistic spectrum sharing in 5G HetNets: A harmonized SDN-enabled approach. IEEE Communications Magazine, 54(1), pp. 40-47.

Balachandran, A.; Sekar, V.; Akella, A. and Seshan, S., 2013. Analyzing the potential benefits of cdn augmentation strategies for internet video workloads. Proceedings of ACM Internet Measurement Conference (IMC), Barcelona, Spain, pp. 43-56.

Begole, B., 2016. Why The Internet Pipes Will Burst When Virtual Reality Takes Off. Forbes. [online] Available at: <http://tinyurl.com/zhvbdjm> [Accessed 20 June 2016].

BGR, 2015. Cable providers still have no answer for Netflix as cord-cutting accelerates. [online] Available at: $<\mathrm{http}: / /$ tinyurl.com/hg2h6fa $>$ [Accessed on 20 June 2016].

Birdstep Technologies, 2016. Product solutions. [online] Available at: $<$ http://www.smithmicro.com/> [Accessed 20 June 2016].

Bridge Technologies, 2015. Bridging With A Virtual Probe For Core Networks. [online] Available at: $<$ http://tinyurl.com/zxyjojz $>$ [Accessed 20 June 2016].

Broadpeak, 2016. NanoCDN multicast ABR. [online] Available at: $<\mathrm{http} / / /$ tinyurl.com/j4hfbv2> [Accessed 20 June 2016].

Bumgardner, G., 2015. Automatic Multicast Tunneling. IETF draft.

Chen, S.; Gao, Z. and Nahrstedt, K., 2016. F.Live: Towards Interactive Live Broadcast FTV Experience. Proceedings of IEEE Conference on Computer Communications (INFOCOM), San Francisco, CA, USA.

Cisco Newsroom, 2016. Coming to a headset near you: The virtual reality revolution. [online] Available at: $<$ http://tinyurl.com/jjkfvut $>$ [Accessed 20 June 2016].

CNET, 2016. Everyone wanted a piece of virtual reality at this year's CES. [online] Available at: $<$ http://tinyurl.com/j81s34g $>$ [Accessed 20 June 2016].

CSI magazine, 2015. New building blocks: Is OTT the future of IPTV? (Sony to trial DVB-T2 to 
mobile in Asia). [online] Available at: <http://tinyurl.com/jxkhryf $>$ [Accessed 20 June 2016].

Dobrian, F.; Sekar, V.; Awan, A.; Stoica, I.; Joseph, D.; Ganjam, A.; Zhan, J.; Zhang, H., 2011. Understanding the impact of video quality on user engagement. SIGCOMM Comput. Commun, 41( 4), pp .362-373.

Elemental Technologies, 2016. Product solutions. [online] Available at: $<\mathrm{http}: / /$ www.elementaltechnologies.com/ $>$ [Accessed 20 June 2016].

Ericsson, 2011. Heterogeneous Networks (HetNet). White paper.

Esteban, J.; Benno, S. A.; Beck, A.; Guo, Y.; Hilt, V. and Rimac, I., 2012. Interactions between HTTP adaptive streaming and TCP. Proceedings of the ACM conference on Network and Operating System Support for Digital Audio and Video (NOSSDAV), Toronto, ON, Canada, pp. 21-26.

ETSI, 2016. Digital cellular telecommunications system (Phase 2+). Technical report [online] Available at: $<$ http://tinyurl.com/hrtfmh4 $>$ [Accessed 20 June 2016].

Free, 2016. Réseau communautaire FreeWiFi_Secure - EAP-SIM. [online] Available at: $<$ http://tinyurl.com/hrer8jj $>$ [Accessed 20 June 2016].

Ganjam, A.; Siddiqui, F.; Zhan, J.; Liu, X.; Stoica, I.; Jiang, J.; Sekar, V.; Zhang, H., 2015. C3: Internet-Scale Control Plane for Video Quality Optimization. Proceedings of USENIX NSDI, Oakland, CA, USA, pp. 131-144.

Georgopoulos, P.; Elkhatib Y.; Broadbent, M.; Mu, M.; Race, N., 2013. Towards network-wide QoE fairness using openflow-assisted adaptive video streaming. Proceedings of ACM SIGCOMM conference, Hong Kong, China, pp. 15-20.

Huffington Post, 2014. Broadcast TV Dead By 2030, Netflix CEO Reed Hastings Says. [online] Available at: <http://tinyurl.com/gn7v4nr> [Accessed 20 June 2016].

IPTV News, 2014. What does the future hold for IPTV? (Lessons learned from Aereo). [online] Available at: $<$ http://tinyurl.com/zg8a834 $>$ [Accessed on 20 June 2016].

IPTV News, 2015. 9/10 demand single app experience for $T V$. [online] Available at: $<$ http://tinyurl.com/zvw5bv3> [Accessed on 20 June 2016].

IPTV news, 2016. 81\% of US households have DVR, Netflix, or VOD. [online] Available at: $<$ http://tinyurl.com/h9odze4 $>$ [Accessed on 20 June 2016].

ISO/IEC DIS 23009-1, 2012. Dynamic adaptive streaming over HTTP (DASH).

Iyengar, J. and Swett, I., 2015. Quic: A UDP-based secure and reliable transport for HTTP/2. IETF draft.

Jiang, J.; Liu, X.; Sekar, V.; Stoica, I.; Zhang, H., 2014. EONA: Experience-Oriented Network 
Architecture. Proceedings of the 13th ACM Workshop on Hot Topics in Networks (HotNets-XIII), Los Angeles, CA, USA.

Karzand, M.; Leith, D. J.; Cloud, J. and Médard, M., 2015. Low delay random linear coding over a stream. Arxiv preprint. Available through: <http://arxiv.org/abs/1509.00167> [Accessed 20 June 2016].

Kim, M; Cloud, J.; ParandehGheibi, A.; Urbina, L.; Fouli, K.; Leith, D. and Medard, M., 2013. Network Coded TCP (CTCP). Arxiv preprint. Available through: $<$ http://arxiv.org/abs/1212.2291> [Accessed 20 June 2016].

Leconte, M. et al, 2016. Placing Dynamic Content in Caches with Small Population. Proceedings of IEEE Conference on Computer Communications (INFOCOM), San Francisco, CA, USA.

Lederer, S.; Posch, D.; Timmerer, C.; Westphal, C.; Azgin, A.; Liu, S.; Muller, C.; Detti, A.; Corujo, C., 2015. Video Streaming over ICN. Internet Draft, ICNRG/IETF.

Lee, K.; Lee, J.; Yi, Y.; Rhee, I. and Chong, S., 2010. Mobile data offloading: how much can WiFi deliver?. Proceedings of ACM International Conference on emerging Networking EXperiments and Technologies (CoNEXT), Philadelphia, PA, USA, pp. 536-550.

Lifehacker, 2014. TV Streaming Head-to-Head: Netflix vs Hulu vs Amazon Prime. [online] Available at: <http://tinyurl.com/jrw2egl> [Accessed 20 June 2016].

Lundin, H.; et al, 2013. Google congestion control algorithm for real-time communication on the world wide web. IETF draft.

Macworld, 2016. Apple TV streaming service release date rumours: CBS CEO says talks with Apple have stopped. [online] Available at: <http://tinyurl.com/ha3vh9c $>$ [Accessed 20 June 2016].

Mintel, 2015. Mintel report. [online] Available at: $<$ http://tinyurl.com/z25gl8t $>$ [Accessed 20 June 2016].

Munford, I., 2016. For the Win! Live Sports Are Driving Streaming Video Innovation. [online] Available at: <http://tinyurl.com/jkq959b $>$ [Accessed 20 June 2016].

Mware Solutions, 2016. Product solutions. [online] Available at: <http://iptvmiddleware.com/> [Accessed 20 June 2016].

Octoshape, 2012. Automatic Multicast Without Tunnels. [online] Available at: $<\mathrm{http} / / /$ tinyurl.com/z5vzhzo $>$ [Accessed 20 June 2016].

Rainer, B.; Posch, D. and Hellwagner, H., 2016. Investigating the Performance of Pull-based Dynamic Adaptive Streaming in NDN. IEEE Journal on Selected in Area on Communications, to appear.

RCR Wireless News, 2015. Ericsson on 5G - HetNet Happenings: Episode 23. [online] Available at: $<$ http://tinyurl.com/hd5sfun $>$ [Accessed 20 June 2016]. 
Sandvine, 2014. Global Internet Phenomena Report. $1^{\text {st }}$ half 2014.

Streaming Media, 2016. The Return of Multicast: Why it Succeeds in a Live Linear World. [online] Available at: <http://tinyurl.com/ze47ssw $>$ [Accessed 20 June 2016].

Streamroot, 2016. Product solutions. [online] Available at: <http://www.streamroot.io/> [Accessed 20 June 2016].

Strategy Analytics, 2015. Chromecast leads global digital media streamer market for fifth straight quarter.[online] Available at: <http://tinyurl.com/z7srjlb $>$ [Accessed on 20 June 2016].

The Telegraph, 2016. BT explores deal with TalkTalk for full control of TV tech. [online] Available at: $<$ http://tinyurl.com/zyvqwg7> [Accessed 20 June 2016].

Time, 2014. Netflix's Disputes With Verizon, Comcast Under Investigation. [online] Available at: $<$ http://tinyurl.com/h7tbez3> [Accessed 20 June 2016].

Verimatrix, 2016. Product solutions. [online] Available at: <http://www.verimatrix.com/> [Accessed 20 June 2016].

WebRTC project, 2016. WebRTC. [online] Available at: <https://webrtc.org/> [Accessed 20 June 2016].

Xylomenos, G.; Ververidis, C.; Siris, V.; Fotiou, N.; Tsilopoulos, C.; Vasilakos, X.; Katsaros, K. and. Polyzos, G., 2014. A Survey of Information-Centric Networking Research. IEEE Communications Surveys and Tutorials, 16(2), pp. 1024-1049.

Yang, P.; Luo, W.; Xu, L.; Deogun, J. and Lu, Y., 2014. TCP congestion avoidance algorithm identification. IEEE/ACM Transactions on Networking, 22(4), pp. 1311-1324.

Yin, X.; Jindal, A.; Sekar, V. and Sinopoli, B., 2015. A Control-Theoretic Approach for Dynamic Adaptive Video Streaming over HTTP. Proceedings of ACM SIGCOMM conference, London, $U K$, pp 325-338.

Yu, Y.; Bronzino, F.; Fan, R.; Westphal, C. and Gerla, M., 2015. Congestion-Aware Edge Caching for Adaptive Video Streaming in Information-Centric Networks. Proceedings of IEEE Consumer Communications \& Networking Conference (CCNC), Las Vegas, NV, USA, pp. 588-596.

Zhao, M.; Aditya, P.; Chen, A.; Lin Y.; Haeberlen, A.; Druschel, P.; Maggs, B.; Wishon, B. and Ponec, M., 2013. Peer-assisted content distribution in Akamai netsession. Proceedings of ACM Internet Measurement Conference (IMC), Barcelona, Spain, pp. 31-42. 